\title{
Action, activité, « agir » conjoints en didactique : discussion théorique
}

Joint Action, Joint Activity, Joint "Acting" in didactics: theoretical discussion

\section{Patrice Venturini}

\section{QpenEdition}

\section{Journals}

\section{Édition électronique}

URL : http://journals.openedition.org/educationdidactique/1348

DOI : 10.4000/educationdidactique.1348

ISSN : $2111-4838$

\section{Éditeur}

Presses universitaires de Rennes

\section{Édition imprimée}

Date de publication : 31 mai 2012

Pagination : 127-136

ISBN : 978-2-7535-1872-8

ISSN : 1956-3485

Référence électronique

Patrice Venturini, «Action, activité, " agir » conjoints en didactique : discussion théorique », Éducation et didactique [En ligne], 6-1 | 2012, mis en ligne le 02 mai 2014, consulté le 09 décembre 2020. URL : http://journals.openedition.org/educationdidactique/1348; DOI : https://doi.org/10.4000/ educationdidactique.1348 


\title{
ACTION, ACTIVITÉ, « AGIR » CONJOINTS EN DIDACTIQUE : DISCUSSION THÉORIQUE
}

\author{
Patrice Venturini (UMR EFTS, Univeristé de Toulouse 2)
}

\begin{abstract}
Résumé : En prolongement d'un article publié par la revue, ce document se propose de discuter l'articulation entre la théorie de l'action conjointe en didactique et la théorie de l'activité proposée par les sciences du travail et de la formation, après qu'elles aient été présentés. La discussion porte d'abord sur la compatibilité entre la théorie de l'action conjointe et les principes généraux qui fondent le système conceptuel sur l'activité. Elle porte ensuite sur la possibilité d'envisager, en écho à l'action conjointe entre le professeur et l'élève, la co-activité entre deux systèmes d'activité (le système d'enseignement et le système d'apprentissage). En plus des perspectives liées à la discussion précédente, la conclusion est l'occasion d'évoquer un autre point de vue articulant action (individuelle) et activité (collective et sociale) pour rendre compte de « l'agir » conjoint didactique.
\end{abstract}

Mots clés : action, activité, théorie de l'action conjointe en didactique, théorie de l'activité, co-activité.

Patrice Venturini

Cross (2010) a publié dans cette même revue Éducation et Didactique un article dans lequel il infère certaines des connaissances professionnelles d'une enseignante de chimie à partir de l'analyse de son action dans plusieurs séances en classe de Terminale. Il mobilise à cette fin la théorie de l'action conjointe en didactique (TACD). En lisant son article, j’ai été interpellé par un chapitre de quelques lignes intitulé «Intentionnalité de l'enseignante » (p. 48), dont le contenu semble d'ailleurs n'avoir été ni repris ni discuté par la suite. Celui-ci fait état lors de la prise de données liée à la recherche réalisée, de l'existence dans la classe de deux types de devoirs, aux dires mêmes de l'enseignante, les devoirs de type «Bac » et les devoirs de type «non Bac». Et l'auteur ajoute: « du point de vue de la TACD, l'existence de ces deux types de sujets peut être interprétée en termes d'intentionnalité de l'enseignante qui cherche à atteindre deux buts: préparer les élèves au Bac et faire en sorte que les élèves apprennent la chimie ». Cette intentionnalité ne s'inscrit pas dans l'immédiateté: réussir le baccalauréat ou maîtriser les concepts de la chimie ne peut s'entendre qu'à moyen terme. Aussi, de mon point de vue, plus que des buts, il s'agit plutôt de perspectives ou de visées pour l'enseignante, auxquelles elle n'est d'ailleurs pas la seule à concourir; les buts en effet sont généralement beaucoup plus proches des actions auxquelles ils sont référés. Aussi, cette " intentionnalité », ces visées à moyen terme, trouveraient mieux il me semble à être interprétées non seulement en termes d'action mais aussi d'activité. La TACD invite d'ailleurs implicitement à ce prolongement, puisqu'elle mentionne la compatibilité du terme d'action (didactique) « avec celui d'activité, au sens de théorie de l'activité (notamment Leontiev, 1984, Clot, 1999)»(Sensevy, 2007, p. 14).

C'est donc la mise en relation entre la TACD et la théorie de l'activité (TA) que cet article se propose de discuter sans bien sûr prétendre clore le débat, bien au contraire, tant il est vrai qu'une réflexion théorique ne prend totalement son sens qu'en se confrontant à la réalité, ce qui ne sera pas le cas ici. Cette réflexion prendra appui sur une brève description de la $\mathrm{TACD}^{1}$ et sur une présentation un peu plus détaillée de la théorie de l'activité. Celles-ci permettront de discuter en quoi et jusqu'où une articulation entre TA et TACD est possible. La conclusion, en plus de tracer des perspectives issues de la discussion précédente, ouvrira sur un point de vue différent à propos de l'articulation entre action et activité.

\section{Un aperçu des deux théories discutées}

\section{Théorie de l'action conjointe en didactique}

Cette présentation s'appuie sur la formalisation proposée par Sensevy (2007). L'action didactique est postulée dans la théorie comme une action conjointe du professeur et de l'élève (id. p. 14). Elle est fondée sur la communication dans la durée entre ces deux types d'acteurs. Professeur et élève sont considérés comme co-acteurs dans une situation qu'ils partagent et qui est centrée sur le savoir à transmettre ${ }^{2}$, même s'ils ont des rôles différents. Aussi leurs interactions sont-elles considérées comme des transactions dont l'objet est le savoir (id. p. 15). L'action didactique liée 
à ces transactions est envisagée comme un « jeu» pour souligner ses aspects affectifs, pragmatiques et effectifs, (id. p. 19), un jeu didactique « organiquement coopératif », que l'on peut décrire de la manière suivante, en considérant les joueurs A et $\mathrm{B}$ : pour gagner au jeu, A doit produire proprio motu certaines stratégies (qui sont gagnantes). B accompagne A dans le jeu, il gagne si A gagne. Connaissant les stratégies à produire, il ne peut toutefois pas les livrer telles quelles à A sous peine de perdre car A ne les produirait pas proprio motu. Ce jeu est décrit par trois ensembles de descripteurs décrits ci-dessous.

- Le doublet contrat-milieu : le contrat didactique correspond à « l'ensemble des comportements (spécifiques) du maître qui sont attendus de l'élève et l'ensemble des comportements de l'élève qui sont attendus du maître » (Sensevy, p. 18 reprenant une définition proposée notamment dans Brousseau et Warfield, 1999, p. 33). Le milieu didactique est « ce qui agit sur l'élève et/ou ce sur quoi l'élève agit » (Brousseau, 2003, p. 3). Il englobe des éléments situationnels locaux et historico culturels, des éléments conceptuels et matériels et des aspects sociaux et sémiotiques (Amade-Escot \& Venturini, 2009). Le milieu constitue à la fois le contexte cognitif et le système antagoniste de l'action. Un jeu didactique est caractérisé par un milieu et un contrat particulier (Sensevy, 2007, p. 23-24).

- Le triplet des genèses: La mésogenèse traduit l'évolution du milieu sous l'action conjointe du professeur et de l'élève et répond à « l'élaboration d'un système commun de significations » (id. p. 30). Le contenu du jeu didactique se modifie continuellement, les jeux se succèdent avec des enjeux de savoir qui évoluent, le savoir avance au cours du temps, c'est la chronogenèse, très liée à la mésogenèse. Enfin, la topogenèse décrit « la répartition des responsabilités dans les transactions didactiques entre professeur et élève » (id. p. 31).

- Le quadruplet définir - dévoluer - régulerinstitutionnaliser: le jeu pour être initié doit d'abord être défini puis il est nécessaire que l'élève accepte de jouer et d'assumer de jouer de manière « adéquate » (dévolution). L'enseignant régule le jeu pendant son déroulement de manière à ce que l'élève parvienne peu à peu à produire proprio motu la stratégie gagnante. Enfin, quand il y parvient, il y a lieu de la reconnaître et de fixer « les manières de faire et penser adéquates au jeu » (institutionnalisation) (id. p. 28).

Le déroulement du jeu dans la classe ainsi décrit dépend fortement des décisions prises dans la phase de préparation. Celles-ci sont à relier au rapport que le professeur entretient avec le savoir en jeu. Mais le déroulement du jeu est aussi lié à des déterminants de nature institutionnelle et épistémologique qui échappent à l'intentionnalité de l'enseignant (id. p. 37). D'une part, l'action didactique est adressée: le professeur ne peut être très longtemps un mauvais sujet des institutions auxquelles il appartient, et son action se conforme donc à leurs exigences. D'autre part, le professeur a des théories implicites sur la nature du savoir enseigné, sur l'enseignement et sur l'apprentissage, et en particulier sur l'enseignement et l'apprentissage du savoir enjeu. Cette « épistémologie pratique » (construite dans la pratique et pour la pratique) contraint aussi l'action didactique.

Décrivons maintenant la théorie de l'activité.

\section{Théorie de l'activité}

L'activité humaine a fait l'objet au cours du temps de diverses approches, philosophiques, sociologiques, psychologiques, ou encore relevant des sciences du travail et de la formation (Bronckart, 2005). Je ne considérerai ici qu'une petite partie des travaux liés aux deux dernières (id., p. 59-80), en référence à la situation de travail et de formation proposée par Cross.

Engeström (1999 p. 1; 2005, p. 59-62) distingue trois générations de théories de l'activité. La première est fondée sur le concept de médiation proposé par Vygotski (1978). Pour celui-ci, l'action humaine est médiée par des artefacts porteurs d'une dimension historique et culturelle qui rattachent la pensée de l'individu à la société dans laquelle il vit ${ }^{3}$. La seconde génération, basée sur les travaux de Leontiev (1981) cesse d'envisager seulement l'individu et prend en compte la nature collective de l'activité. Relayée en occident et formalisée en particulier par Engeström (1987), la théorie envisage l'activité comme un système associant l'individu et la communauté à laquelle il appartient, et permet d'analyser comment sont atteints les objectifs collectifs. Enfin, une troi- 
sième génération de travaux est en cours de développement qui cherche « to develop conceptual tools to understand dialogue, multiple perspectives and networks of interacting activity systems » (Engeström, 2005, p. 62). Il s'agit ici d'analyser l'activité collective (conjointe) résultant de la mise en relation de plusieurs systèmes d'activité.

Avant de développer brièvement la formalisation d'Engeström qui sert de base à la plupart des travaux actuels sur l'activité, précisons que la TA n'est pas une théorie au sens strict du terme. Il s'agit plutôt de $"$ a set of basic principles which constitute a general conceptual system which can be used as a foundation for more specific theories » (Bannon, 1997). Selon Kaptelinin, (1996, p. 107-110), ces principes sont au nombre de six: " unity of conscioussness and activity, object-orientedness, hierarchical structure of activity, duality internalisation - externalisation, tool mediation, continuous development $»$.

1. «Unity of conscioussness and activity»: la conscience (dans le sens de connaissance) émerge des interactions signifiantes entre l'individu et son environnement. Nous sommes donc éloignés d'une conception strictement «mentaliste » de la connaissance, ce principe affirmant que « the human mind emerges and exists as a special component of human interaction with the environment »

2. «Object-orientedness » : l'environnement est considéré tant dans sa dimension objective traditionnelle (au sens des sciences de la nature) que dans ses dimensions sociales et culturelles.

3. "Hierarchical structure of activity»: ce principe renvoie à la différenciation faite par Léontiev (1981, p. 59-69) entre

- $\quad$ les activités, orientées par un mobile, objet matériel(s) ou idéel(s) satisfaisant un besoin (le besoin « motive » l'activité);

- les actions, subordonnées aux activités, orientées par des buts conscients;

- les opérations, permettant de réaliser les actions, déterminées par les conditions de l'activité au moment où elles sont mises en œuvre. Il s'agit de comportements souvent routinisés et dont les buts ne relèvent pas du même niveau de conscience que les actions.

Ainsi, Leontiev distingue les mobiles de l'activité humaine des buts vers lesquels l'action qui y participe est immédiatement dirigée. Par ailleurs, une activité donne lieu à un ensemble d'actions, une action peut participer à diverses activités.

4. "(Duality) internalisation - externalisation»: ce principe introduit par Vygotski (1978) décrit le mécanisme à l'origine des processus mentaux. Ces derniers sont considérés comme dérivant de l'internalisation d'actions externes conduites en coopération avec d'autres individus: "consciousness and meaning are always formed in joint, collective activity » (Miettinen 1997, citant Leontjev, 1978²). Les processus mentaux se manifestent eux-mêmes dans des actions externes qui peuvent être vérifiées et corrigées si nécessaires. «Activity Theory emphasizes that internal activities cannot be understood if they are analyzed separately, in isolation from external activities, because there are mutual transformations between these two kinds of activities » (Bannon, 1997).

5. « (Tool) mediation »: la médiation par les outils (externes comme les outils matériels, ou internes comme les concepts ou les systèmes symboliques) tient une place centrale dans les théories de l'activité, notamment en raison de l'importance donnée aux facteurs sociaux et aux interactions entre acteurs et environnements. "The use of these culture-specific tools shapes the way people act and, through the process of internalization, greatly influences the nature of mental development. Tools are thus the carriers of cultural knowledge and social experience " (Kaptelinin, 1996, p. 109).

6. « (Continuous) development $»$ : les interactions entre les êtres humains et leur environnement sont analysées en termes de développement: "activity Theory sees all practices as a result of certain historical developments under certain conditions and as continuously re-forming and developing processes » (Bannon, 1997).

Ces principes ne sont pas isolés, ils sont interreliés et doivent être considérés comme un système intégré d'éléments indissociables. La modélisation de l'activité que propose Engelström (figure 1) et qui est fréquemment utilisée, en reprend la totalité. 


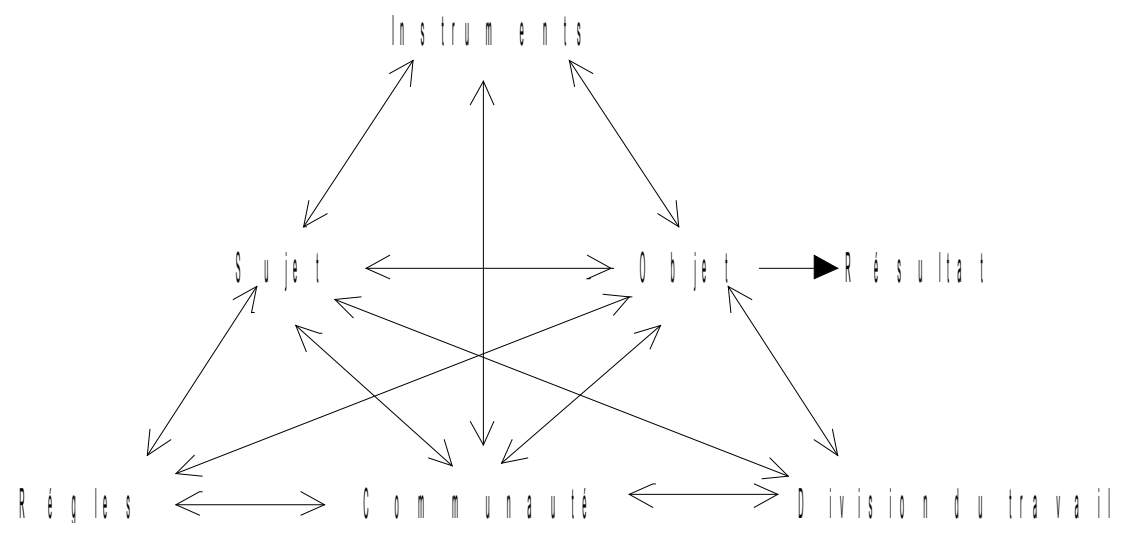

Figure 1: Modélisation de l'activité, d'après Engeström (1987, p. 78; 2005 p. 30)

Dans ce modèle, le sujet réfère à l'acteur (ou au groupe d'acteurs) impliqué(s) dans l'activité et dont on prend le point de vue. L'objet constitue la cible, la visée de l'activité et l'oriente en vue de l'obtention d'un certain résultat. Pour y parvenir, l'acteur a à sa disposition des instruments, artefacts matériels ou symboliques (dont le langage), qui vont assurer une médiation entre le sujet et l'objet de l'activité. La transformation de l'objet de l'activité en résultat constitue le moteur (mobile) de l'activité. Dans cette perspective, le sujet est rarement seul, il fait partie de la communauté de personnes dont l'activité a un objet identique (communauté d'intérêts, de pratiques ou de culture). Aussi, Engeström propose deux autres formes de médiation: d'une part les règles explicites ou tacites (normes, conventions, procédures administratives, pratiques de travail, relations sociales, qui constituent un « héritage culturel » propre à la communauté) médient les interactions entre sujet et communauté; d'autre part, la division du travail (organisation que la communauté se donne pour satisfaire l'objet de l'activité en distribuant les rôles et les responsabilités) médie les relations entre sujet et objet de l'activité. Une telle représentation de l'activité permet de saisir simultanément le travail d'un individu ou d'un collectif restreint et son inscription dans une organisation.

L'ensemble de ces composantes et leurs interactions constituent un système d'activité, «which is taken as the prime unit of analysis against which scripted strings of goal-oriented actions and auto- matic operations are interpreted " (Engeström, 2000, p. 960). Considéré donc comme unité d'analyse de l'activité (Cole \& Engeström, 1993, p. 8), ce système « connects the psychological, cultural and institutional perspective to analysis. The study of activity ceases to be psychology of an individual but instead focuses on the interaction between an individual, systems of artefacts and other individuals in historically developing institutional settings » (Miettinen, 1997). Ces différents éléments s'influencent les uns les autres, font l'objet de contradictions internes ${ }^{5}$, entrent éventuellement en contradiction les uns avec les autres ou avec d'autres systèmes d'activité, amenant continuellement ajustements et évolutions. Le système d'activité est donc dynamique et permet le développement des acteurs et des pratiques: « the systemic contradictions, manifested in disturbances and mundane innovations, offer possibilities for expansive developmental transformation $»$ (Engeström, 2000, p. 960).

Les systèmes d'activité ne sont pas isolés les uns des autres et pour mieux comprendre ce qu'il se passe au sein de l'un d'entre eux, on peut examiner les interactions qu'il développe avec ses voisins (Engeström, 2005, p. 62-63). Cet examen apporte souvent un éclairage complémentaire mais devient indispensable quand plusieurs systèmes sont associés dans un travail identifié comme conjoint ${ }^{6}$. Pour comprendre ce qui se joue lors des interactions entre ces systèmes, Engeström développe trois concepts principaux. Le « boundary crossing » (Engeström, Engeström \& Karkkainen, 1995, p. 320-322) 
recouvre les processus (peu étudiés) en jeu dans ces interactions et pourrait être analysé « as a process of collective concept formation » (id., p. 321). Deux de ces processus, le « knotworking » (travail en nœud) et la « co-configuration », ont fait l'objet d'analyses. "The notion of knot refers to rapidly pulsating, distributed and partially improvised orchestration of collaborative performance between otherwise loosely connected actors and activity system » (Engeström, 2000, p. 972). Dans ce cadre, les fils de l'activité sont noués, dénoués, renoués, le centre de l'initiative évoluant au cours du temps et ne pouvant être rattaché à un individu ou un groupe particulier. L'objectif est ici de résoudre un problème temporaire, les frontières entre les systèmes étant franchies de manière fugace même si la collaboration est inscrite dans le temps. La co-configuration $^{7}$ (Engeström, 2000, p. 972-974; 2004, p. 11-13) constitue une nouvelle forme de travail dans laquelle un fabricant développe dans la durée un partenariat interactif avec ses fournisseurs et ses clients pour concevoir, en les co-configurant, des produits ou des services complexes qui évoluent régulièrement dans le temps. Selon Engeström (2000, p. 973), « knotworking may be seen as the emerging interactional core of co-configuration ».

Le cadre de la théorie de l'activité étant sommairement brossé, mettons-le en relation avec celui de la TACD.

\section{Discussion: quelle articulation entre TACD et TA?}

\section{Relations entre TACD et les principes fondant la TA}

Tout d'abord, on peut reconnaître avec Sensevy (2007, p. 36, note 4) une certaine compatibilité entre ces deux cadres théoriques, dans la mesure où tous deux partagent selon lui une même conception épistémologique de l'action humaine, non mentaliste et reconnaissant « l'importance du social dans la production des pensées et des conduites ». On retrouve en effet ces éléments très nettement affirmés dans les deux premiers principes évoqués plus haut, «Unity of conscioussness and activity " et « Objectorientedness ». Dans le même esprit, on peut rapprocher la médiation de l'activité par les outils matériels et symboliques chargés d'une valence historique, culturelle et sociale (principe 5) du rôle important attribué dans l'action didactique conjointe au milieu. Celui-ci renvoie selon la TACD « à l'environnement cognitif commun... et aux ressources et contraintes qui orientent les transactions dans le jeu hic et nunc » (id. p. 23), et selon Amade-Escot et Venturini (2009), il comporte notamment les mêmes dimensions sociales, historiques et culturelles. Enfin, même si la TACD ne mentionne pas directement l'idée de «continuous development " (principe 6), celle-ci est tout de même présente. La TACD suppose en effet implicitement le développement de l'élève auquel le professeur est censé transmettre un savoir entendu au sens anthropologique de puissance d'action sur le monde (Sensevy, 2007 p. 14). Elle envisage aussi le développement de l'enseignant dont l'action didactique permet de construire en grande partie l'épistémologie pratique (id. p. 38), même si celle-ci reste généralement implicite. De plus, la TACD reprend à son compte l'idée que la compréhension d'un phénomène (didactique) passe par la connaissance de la manière dont il se développe dans sa forme existante, localement à travers les aspects chrono, topo et mésogénétiques (id. p. 30-32), plus largement en considérant l'action didactique comme inscrite dans une institution (id. p. 37) et donc liée à son histoire.

Par contre, on ne retrouve pas me semble-t-il dans la TACD, d'équivalence au principe 4 décrivant les processus mentaux sous la forme d'une dualité internalisation - externalisation, mais cette théorie n'a pas pour ambition de décrire le processus d'apprentissage de l'élève. Cependant, la manière dont elle rend compte du volet « externe » me paraît s'inscrire dans une même filiation considérant que le savoir et le sens sont construits dans le cadre d'une activité collective et conjointe.

Il reste enfin à s'interroger sur la hiérarchisation faite par Leontiev entre activité, action et opération (principe 3). Les «actions» qui sont identifiées dans la TACD sont des actions méso, chrono, et topo génétiques. Elles peuvent être considérées tantôt comme des opérations tantôt comme des actions, au sens de la théorie de l'activité, en fonction du contexte et aussi de l'expérience de l'enseignant ${ }^{8}$. Par ailleurs, raisonner en termes d'activité conduit aussi à faire un choix temporel, les échelles de temps envisagées pouvant aller (à l'extrême) de la durée d'un jeu didactique à celle de la scolarité toute entière (si 
l'on considère que la seule activité menée à l'école a pour objet l'insertion de l'élève dans le monde) ${ }^{9}$. Le système des actions et des opérations subordonnées à ces deux types d'activité et dont il faut rendre compte n'a pas la même ampleur et ne recèle pas la même complexité dans les deux cas. Articuler TACD et TA implique donc de préciser et d'articuler dans les analyses à la fois l'activité considérée et ses mobiles ainsi que tout ou partie des actions qui y concourent et les buts qu'elles poursuivent; cela implique aussi de distinguer ces actions d'opérations plus automatisées et plus élémentaires. Ce type d'analyse conduit ainsi à construire un système descriptif et explicatif beaucoup plus complet mais beaucoup plus complexe à construire.

\section{Action conjointe et co-activité résultat de deux systèmes d'activité en interaction}

Si on reprend la définition du jeu didactique dans la TACD (id. p. 20) qui a déjà été évoquée, l'élève gagne quand il produit certaines stratégies « proprio motu » et le professeur gagne lorsque l'élève « produit les stratégies gagnantes (raisonnablement) proprio motu ». L'action didactique conjointe aboutit donc à un seul et même résultat que l'on prenne le point de vue du professeur ou celui de l'élève, la production des stratégies gagnantes par l'élève. Cela ne veut pas dire pour autant comme le font remarquer Schubauer-Leoni et al. (2007, p. 56) que les buts poursuivis par les élèves et le professeur sont vraiment partagés: " plus qu'un partage de buts communs, c'est plutôt une interdépendance entre des buts distincts dont il s'agit ». En écho à cette analyse et en se référant à la troisième génération des théories de l'activité, on peut considérer ce résultat issu de l'action conjointe comme inscrit dans un ensemble plus vaste constitué par l'interaction de deux systèmes d'activité nécessaires pour atteindre conjointement une visée commune ${ }^{10}$, par exemple la maîtrise de la chimie de Terminale par l'élève.

- Le premier système contribue à la visée commune par l'enseignement de la chimie, et le sujet du système envisagé est le professeur. On peut considérer ce dernier comme appartenant par exemple à la communauté des professeurs chargés d'enseigner la chimie aux élèves de l'établissement (ou encore à celle des professeurs enseignant la chimie dans les classes de Terminale). Dans chacun des cas, il faudra alors préciser les règles propres à la communauté concernée ainsi que la division du « travail » d'enseignement de la chimie entre les différents individus ou groupes, tout comme les instruments divers utilisés par le sujet et la communauté pour parvenir à transformer l'objet de l'activité en résultat.

- Le second système contribue à la visée commune par l'apprentissage de la chimie. Le sujet est un élève ou un groupe d'élève (par exemple un petit groupe, ou encore la classe) qui appartient à une communauté (les élèves d'une classe de chimie de Terminale). Dans ce cas aussi, il convient d'identifier les règles communautaires, la division du travail et les instruments dont une partie est d'ailleurs commune avec le système précédent.

La visée commune permet de définir les systèmes en interaction. Toutefois, si la co-activité des deux systèmes correspond à une visée commune, il n'y a pas plus d'unicité des mobiles visés par l'activité des sujets des deux systèmes (professeur et élève(s)) qu'il n'y a d'unicité des buts de leurs actions en classe.

Si l'on adopte ce point de vue, il s'agira alors d'observer comment chaque sujet parvient à réaliser l'objet de son activité au sein de son système d'activité en interagissant aux frontières avec le système partenaire. Il s'agira ainsi d'envisager jusqu'où la collaboration momentanée du professeur avec deux ou trois élèves, puis avec deux ou trois autres, puis la collaboration entre quelques élèves par exemple pour faire avancer le savoir dans la classe peut s'apparenter à du « knotworking », d'envisager quel rôle ce processus tient dans l'interaction, ou encore en quoi et jusqu'où le résultat obtenu apparaît comme co-configuré. Un exemple de ce type d'étude est proposé par Owen (2008) dans un tout autre domaine. Elle analyse en effet la co-activité de deux systèmes d'activité, celui des pilotes d'une compagnie aérienne et celui du contrôle aérien, qui visent tous deux à poser un avion dans les conditions de sécurité requises.

La mise en relation avec la TACD de cette modélisation de l'enseignement/apprentissage par des interactions entre deux systèmes d'activité différents et complémentaires nécessiterait aussi d'identifier en quoi et jusqu'où le contrat et le milieu didactiques, 
voire la dévolution utiles pour comprendre l'action didactique pourraient trouver des équivalents dans la co-activité didactique des deux systèmes, en quoi et comment ces équivalents pourraient permettre le «boundary-crossing » et le travail aux frontières, en quoi ils peuvent être rattachés aux règles, aux communautés et aux instruments propres à chaque système d'activité.

\section{Conclusion}

Le cœur de la TACD a été bâti sur des travaux de la didactique francophone eux-mêmes inscrits dans les mêmes paradigmes que ceux qui ont supporté le développement de la TA (paradigmes faisant par exemple une part importante au contexte et au social). Cette situation explique la compatibilité que l'on a pu relever entre théorie de l'action conjointe et théorie de l'activité. Elle confère aussi à la TACD une certaine densité théorique dans la mesure où l'action dont elle rend compte englobe à la fois des éléments locaux, situés, et des éléments reliés à l'histoire et à la culture des institutions dans lesquelles l'action se développe, ces éléments étant répartis entre opération, action et activité dans la théorie de l'activité.

Toutefois, il reste à voir ce que l'on peut faire de cette compatibilité. L'intérêt d'une théorie réside notamment dans sa possibilité de produire des modélisations expliquant, interprétant le réel (voire le prévoyant, mais on n'en est pas encore là en didactique). De ce point de vue-là, la TACD remplit son rôle, même si certains de ses éléments (les « déterminants " de l'action par exemple) méritent de mon point de vue d'être affinés. Il reste donc à voir maintenant en quoi la modélisation du réel didactique par l'association de deux systèmes d'activité en interaction permet d'en rendre compte différemment ou non de la TACD et plus pertinemment ou non (pour des pistes méthodologiques sur l'analyse d'un système d'activité voir par exemple Sandars, 2005, p. 195-197). Cette phase doit permettre d'apprécier plus finement et de formaliser les zones de recouvrement entre les deux théories. Elle doit aussi permettre de juger de l'intérêt qu'il y aurait ou non à les utiliser simultanément au cours d'une étude, en prolongement l'une de l'autre ou en superposition, à propos d'un même élément du réel.
En attendant d'avoir mené ces travaux, on peut considérer avec intérêt la proposition Bronckart (2005) qui, après avoir passé en revue un vaste ensemble de théories de l'action conclue son ouvrage en proposant (p. 82) une articulation entre action et activité différente de celle que l'on peut trouver dans la théorie de l'activité. Tout d'abord, pour éviter les confusions dans le vocabulaire, il évoque «l'agir » pour désigner (id. p. 81) «toute forme d'intervention orientée d'un ou plusieurs humains dans le monde », l'agir pouvant constituer un travail dont la structure peut être décomposée en tâches, et temporellement, une chaîne d'actes ou de gestes. Pour Bronckart, l'activité désigne « une lecture de l'agir impliquant les dimensions motivationnelles et intentionnelles mobilisées au niveau collectif », et l'action correspond à « une lecture de l'agir impliquant les mêmes dimensions mobilisées au niveau des personnes singulières $»$. Il distingue sur le plan motivationnel « les déterminants externes d'origine collective qui peuvent être de nature matérielle ou de l'ordre des représentations et les motifs qui sont les raisons d'agir telles qu'elles sont intériorisées pas une personne singulière ». Il opère une distinction semblable au niveau de l'intentionnalité, considérant « les finalités d'origine collective et socialement validées et les intentions en tant que fins de l'agir telles qu'elles sont intériorisées par une personne singulière. » L'activité renverrait donc selon Bronckart à du collectif, du social et l'action à de l'individuel, les deux aspects étant articulés. Si l'on emprunte à Schubauer-Leoni et al. (2007, p. 56) l'idée d'un « agir dans le cas spécifique, des interactions didactiques et des transactions qui les caractérisent » que l'on pourrait désigner plus simplement par « agir didactique ", la « théorie de l'agir conjoint en didactique » (et non plus de l'action pour éviter les confusions de vocabulaire) peut rendre compte des dimensions «action » et « activité » de cet agir telles que définies plus haut, la première prenant en compte à la fois l'individu comme être singulier et le contexte local en les incluant dans une temporalité très courte, la seconde prenant en compte l'individu comme être social appartenant à différentes communautés et institutions en l'incluant dans une temporalité beaucoup plus longue. Tenter de structurer la TACD de la sorte (où A serait l'initiale d'agir) aurait certainement de l'intérêt pour rendre plus lisibles les phénomènes didactiques. 


\section{NOTES}

1. La description est brève parce que cette théorie a été fréquemment développée dans la revue.

2. En considérant transmettre dans le sens anthropologique général (p. 14)

3. La médiation «breaks down the Cartesian walls that isolate the individual mind from the culture and society » (Engeström, 1999 p. 29).

4. Leont'ev, A.N. 1978. Activity, consciousness and personality. Englewood Cliffs, NJ : Prentice Hall.

5. Par exemple, lorsqu'il analyse l'évolution culturelle de l'apprentissage humain, Engeström (1987, p. 103) relève que l'on peut envisager la communauté comme un ensemble d'individus isolés vs une équipe de "recherche ", les instruments comme des outils de mémorisation et procéduralisation vs les outils d'investigation, le sujet comme cherchant à obtenir un diplôme vs cherchant à produire du sens, etc.

6. Par exemple on peut imaginer un système d'activité associé au staff technique et un autre associé au staff médical, dont le travail conjoint a pour objectif d'amener un athlète au sommet de son art à un moment précis de la saison.

7. Ce concept a été emprunté par Engeström (2000, p. 972, 2004, p. 11) à Victor et Boynton (Victor, B. and Boynton, A. C. (1998). Invented Here: Maximising Your Organisation's Internal Growth and Protability. Boston: Harvard Business School Press.)

8. Ce qui peut être envisagé comme action chez un enseignant débutant, c'est-à-dire relié à des buts identifiés, peut être considéré comme opération chez un enseignant expérimenté qui l'aurait routinisée.

9. On retrouve les questions d'échelle d'analyse micro, méso et macro pointées dans les études de Tiberghien (par exemple: Tiberghien, A., Malkoun L., Buty, C., Souassy, N. \& Mortimer E. (2007). Analyse des savoirs en jeu en classe de physique à différentes échelles de temps. In G. Sensevy \& A. Mercier (Ed.), Agir ensemble. L'action didactique conjointe du professeur et des élèves (p. 73-98). Rennes: Presses Universitaires de Rennes.)

10. Si on se situe en dehors d'une action didactique conjointe professeur - élève, probablement peut-on interpréter des phénomènes liés à l'enseignement et l'apprentissage à partir d'un seul système d'activité où le sujet est l'enseignant (voir par exemple Benayed \& Verreman, 2006; Lantolf \& Genung, 2000) mais ce n'est pas notre propos ici.

\section{RÉFÉRENCES}

Amade-Escot, C., \& Venturini, P. (2009). Le milieu didactique: d'une étude empirique en contexte difficile à une réflexion sur le concept. Éducation et Didactique, 3 (1), p. 7-43.

Bannon, L. (1997). Activity Theory. Retrieved on February 01 2011, from http://www.irit.fr/ACTIVITES/GRIC/ $\operatorname{cotcos/pjs/TheoreticalApproaches/Actvity/ActivitypaperBannon.htm~}$

Benayed, M., \& Verreman, A. (2006). Évaluation d'un dispositif d'apprentissage collaboratif à distance. Informations Savoirs Décisions \& Médiations, 25. Retrieved on February 01 2011, from http://isdm.univtln.fr/PDF/isdm25/BenayedVerreman_TICE2006.pdf 
Bronckart, J.-P. (2005). Une introduction aux théories de l'action. Genève: université de Genève, FPSE Publications.

Brousseau, G. \& Warfield, V. (1999). The case of Gaël The study of a child with mathematical difficulties. The Journal of Mathematical Behavior, 18 (1). Retrieved on February 012011 from http://daest.pagespersoorange.fr/guy-brousseau/textes/CasdeGael.pdf

Brousseau, G. (2003). Glossaire de quelques concepts de la théorie des situations didactiques en mathématiques. Retrieved September $1^{\text {st }} 2010$ from http://pagesperso-orange.fr/daest/guy-brousseau/textes/Glossaire_ Brousseau. pdf

Chevallard, Y. (1992). Concepts fondamentaux de la didactique: perspective apportée par une approche anthropologique. Recherches en Didactique des Mathématiques, 12 (1), p. 73-112.

Chevallard, Y. (2003). Approche anthropologique du rapport au savoir et didactique des mathématiques. In S. Maury \& M. Caillot (dir.), Rapport au savoir et didactiques (p. 81-122). Paris: Fabert.

Cole, M., \& Engestrom, Y. (1993) A cultural-historical approach to distributed cognition. In G. Salomon (Ed.), Distributed cognitions: Psychological and educational considerations. New York: Cambridge University Press (p. 1-46)

Cross, D. (2010). Action conjointe et connaissances professionnelles de l'enseignant. Éducation et Didactique, 4 (3), p. 31-54.

Engeström, Y. 1987. Learning by expanding. An activity theoretical approach to developmental research. Helsinki: Orienta Konsultit.

Engeström, Y. (1999). Learning by expanding: Ten years after. Introduction to the German edition of Learning by Expanding, published under the title Lernen durch Expansion: Marburg: BdWi-Verlag. Retrieved February 01 2011, from http://lchc.ucsd.edu/MCA/Paper/Engestrom/expanding/intro.htm

Engeström, Y. (2000). Activity theory as a framework for analyzing and redesigning work. Ergonomics, 43 (7), p. $960-974$.

Engeström, Y. (2004). New forms of learning in co-configuration work. Journal of Workplace Learning, 16, p. 11-21

Engeström, Y. (2005). Developmental work research: expanding activity theory into practice. Berlin: Lehmanns Media.

Engeström, Y., Engeström, R., \& Karkkainen, M. (1995). Polycontextuality and boundary crossing in expert cognition: learning and problem solving in complex work activities. Learning and Instruction, 5, 319-336.

Kaptelinin, V. (1996). Activity Theory: Implications for human-computer interaction. In B. Nardi, (Ed.), Context and Consciousness: Activity theory and human-computer interaction. Cambridge, MA: MIT Press

Lantolf, J.-P., \& Genung, B. (2000). L'acquisition scolaire d'une langue étrangère vue dans la perspective de la théorie de l'activité: une étude de cas. Acquisition et Interactions en Langue Étrangère, 12. Retrieved on February 01 2011, from http://aile.revues.org/document280.html. 
Leontiev, A. N. (1981). The problem of activity in psychology. In J.V.Wertsch (ed.), The concept of activity in Soviet psychology. Armonk, NY: M. E. Sharpe.

Miettinen, R. (1997, july), The Concept of Activity in the Analysis of Heterogeneous Networks in Innovation Process. "Actor Network and After », Centre for Social Theory and Technology Workshop: University of Helsinki. Retrieved on February 01 2011, from http://communication.ucsd.edu/MCA/Paper/Reijo/Reijo. html\#Introduction

Owen, C. (2008). Analyser le travail conjoint entre différents systèmes d'activité. Activités, 5 (2), p. 70-89. Retrieved on February 01 2011, from http://www.activites.org/v5n2/owen-FR.pdf

Sandars, J. (2005). An activity theory perspective. Work Based Learning in Primary Care, 3, p. 191-201.

Schubauer-Leoni, M., Leutenegger, F., Ligozat, F. \& Fluckinger, A. (2007). Un modèle de l'action conjointe professeur-élèves: les phénomènes didactiques qu'il peut/doit traiter. In G. Sensevy \& A. Mercier, (2007) (dir.), Agir ensemble: l'action didactique conjointe du professeur et des élèves (p. 51-91). Rennes: Presses Universitaires de Rennes.

Sensevy, G. (2007). Des catégories pour décrire et comprendre l'action didactique. In G. Sensevy \& A. Mercier, (2007) (dir.), Agir ensemble: l'action didactique conjointe du professeur et des élèves (p. 13-49). Rennes: Presses Universitaires de Rennes

Vygotsky, L. (1978 / 1930). Mind in society: The development of higher psychological processes. Cambridge, MA: Harvard University Press. 